\title{
Acetaminophen for analgesia following pyloromyotomy: does the route of administration make a difference?
}

\author{
This article was published in the following Dove Press journal: \\ Journal of Pain Research \\ 8 March 2016 \\ Number of times this article has been viewed
}

\author{
Arvid Yung' \\ Arlyne Thung ${ }^{1,2}$ \\ Joseph D Tobias ${ }^{1-3}$ \\ 'Department of Anesthesiology \\ and Pain Medicine, Nationwide \\ Children's Hospital, ${ }^{2}$ Department of \\ Anesthesiology and Pain Medicine, \\ ${ }^{3}$ Department of Pediatrics, The Ohio \\ State University College of Medicine, \\ Columbus, OH, USA
}

Background: During the perioperative care of infants with hypertrophic pyloric stenosis, an opioid-sparing technique is often advocated due to concerns such as postoperative hypoventilation and apnea. Although the rectal administration of acetaminophen is commonly employed, an intravenous (IV) preparation is also currently available, but only limited data are available regarding IV acetaminophen use for infants undergoing pyloromyotomy. The objective of the current study was to compare the efficacy of IV and rectal acetaminophen for postoperative analgesia in infants undergoing laparoscopic pyloromyotomy.

Methods: A retrospective review of the use of IV and rectal acetaminophen in infants undergoing laparoscopic pyloromyotomy was performed. The efficacy was assessed by evaluating the perioperative need for supplemental analgesic agents, postoperative pain scores, tracheal extubation time, time in the postanesthesia care unit, time to oral feeding, and time to hospital discharge.

Results: The study cohort included 68 patients, of whom 34 patients received IV acetaminophen and 34 received rectal acetaminophen. All patients also received local infiltration of the surgical site with $0.25 \%$ bupivacaine. No intraoperative opioids were administered. There was no difference between the two groups with regard to postoperative pain scores, need for supplemental analgesic agents, time in the postanesthesia care unit, or time in the hospital. There was no difference in the number of children who tolerated oral feeds on the day of surgery or in postoperative complications.

Conclusion: Our preliminary data suggest that there is no clinical difference or advantage with the use of IV versus rectal acetaminophen in infants undergoing laparoscopic pyloromyotomy.

Keywords: postoperative pain management, neonatal anesthesia, pyloric stenosis, pain score, length of hospital stay, length of postanesthesia care unit stay

\section{Introduction}

Pyloric stenosis (PS) is one of the most common surgical conditions affecting infants with an incidence of approximately $0.9 \%-5.1 \%$ per 1,000 live births, generally presenting prior to 3 months of life. ${ }^{1,2}$ Since the end of the 19th century, surgical intervention has been the primary treatment with first open and then subsequently laparoscopic pyloromyotomy. ${ }^{3}$ During the postoperative period, various options exist for the provision of postoperative analgesia, including opioids and non-opioid analgesics such as acetaminophen, local infiltration of the surgical site, or regional anesthesia. Given the concerns of respiratory depression related to the patient's age and metabolic abnormalities, the use of opioids is generally limited or avoided. ${ }^{4-9}$ The current study retrospectively investigates the impact of the route of administration of acetaminophen
Correspondence: Arvid Yung

Department of Anesthesiology and Pain Medicine, Nationwide Children's Hospital, 700 Children's Drive, Columbus, $\mathrm{OH}$ 43205, USA Email Arvid.Yung@Nationwidechildrens. org 
(intravenous [IV] versus rectal) on the analgesic effect following pyloromyotomy in infants.

\section{Methods}

This study was approved by the Institutional Review Board of Nationwide Children's Hospital which deemed the study scientific and ethical (IRB 14-00149), and registered at clinicaltrials.gov (NCT02359305). The Institutional Review Board of Nationwide Children's Hospital waived the need to obtain written informed consent from the patients due to the retrospective nature of this study. Infants undergoing pyloromyotomy over the past 3 years were identified and their records were retrospectively reviewed. Patients who received opioids intraoperatively were excluded. Patient demographic data included age at the time of surgery, weight, and sex. Surgical information included surgical time and the type of procedure (open versus laparoscopic). The intraoperative anesthetic record was reviewed, and the following data were obtained: the route (IV or rectal) of administration and the dose of acetaminophen, additional medications that were administered intraoperatively including opioids or nonsteroidal anti-inflammatory agents, and the use of local anesthetic infiltration of the surgical site. Postoperative variables included the place of tracheal extubation (operating room [OR] or postanesthesia care unit [PACU]), pain scores, time in the PACU, the need for supplemental analgesic agents in the PACU and during the subsequent postoperative period, time to oral feeding, and time to hospital discharge. As per institutional routine, postoperative surgical pain is assessed in infants using the Faces, Leg, Activity, Cry, and Consolability scale, which assigns a score of $0-10 .{ }^{10}$ To examine whether there were any statistical significant differences between the two groups (rectal and IV acetaminophen), a Fisher's exact test was performed for the categorical variables. With the exception of weight, the continuous variables were nonnormally distributed; therefore, a Wilcoxon rank sum test was performed to examine the differences, while an independent sample $t$-test with an equal variance assumption was performed for weight. The data are presented as the mean \pm standard deviation with $P<0.05$ considered significant.

\section{Results}

A total of 80 patients were identified who had undergone a pyloromyotomy for PS, of which 12 patients were excluded from the study as they received intraoperative opioids. This resulted in a final study cohort of 68 infants. All the patients had a laparoscopic approach for pyloromyotomy. There were
Table I Cohort demographics

\begin{tabular}{lll}
\hline Characteristic & $\begin{array}{l}\text { Intravenous } \\
\text { acetaminophen }\end{array}$ & $\begin{array}{l}\text { Rectal } \\
\text { acetaminophen }\end{array}$ \\
\hline Weight $(\mathrm{kg})$, mean \pm SD & $4.0 \mathrm{I} \pm 0.80$ & $3.87 \pm 0.7 \mathrm{I}$ \\
Age (days), mean \pm SD & $34.2 \pm 14.5$ & $37.8 \pm 16.5$ \\
Sex (male/female), $\mathrm{n}$ & $29 / 5$ & $29 / 5$ \\
\hline
\end{tabular}

34 patients who received IV acetaminophen and 34 who received rectal acetaminophen. The demographics of the two groups are listed in Table 1. The dose of IV acetaminophen was $8.6 \pm 3.9 \mathrm{mg} / \mathrm{kg}$, while the dose of rectal acetaminophen was $30.7 \pm 6.3 \mathrm{mg} / \mathrm{kg}$. All the patients also received local infiltration of the surgical site with $0.25 \%$ bupivacaine at the completion of the procedure. No patients received intraoperative opioids or other analgesic agents in the OR. Two patients received opioids during the postoperative course. Both were in the rectal acetaminophen group. One received a dose of fentanyl in the PACU, and another received a dose of morphine in the inpatient ward. There was no difference between the two groups with regard to pain scores, time spent in the PACU, or time spent in the hospital (Table 2). PACU pain scores, final PACU pain score, and average postoperative pain scores were similar in both groups (Table 1). As per our clinical practice, depending on their emergence from anesthesia, the trachea of patients is either extubated in the OR or in the PACU. There was no difference in the site of tracheal extubation between the two groups. Twenty-four of 34 patients who received IV acetaminophen had their tracheas extubated in the OR compared with 21 of 34 who received rectal acetaminophen. There was no difference in the number

Table 2 Postoperative outcomes and pain scores

\begin{tabular}{lll}
\hline Outcome & $\begin{array}{l}\text { Intravenous } \\
\text { acetaminophen }\end{array}$ & $\begin{array}{l}\text { Rectal } \\
\text { acetaminophen }\end{array}$ \\
\hline $\begin{array}{l}\text { Average FLACC pain score in } \\
\text { the PACU }\end{array}$ & $0.3 \pm 0.6$ & $0.6 \pm \mathrm{I}$ \\
$\begin{array}{l}\text { Final FLACC pain score in the } \\
\text { PACU }\end{array}$ & $0.0 \pm 0.0$ & $0.1 \pm 0.3$ \\
$\begin{array}{l}\text { Initial postoperative FLACC } \\
\text { pain score in the ward }\end{array}$ & $0.6 \pm 1.6$ & $1.6 \pm 3.0$ \\
$\begin{array}{l}\text { Average postoperative FLACC } \\
\text { pain score }\end{array}$ & $0.5 \pm 0.5$ & $0.5 \pm 0.5$ \\
$\begin{array}{l}\text { PACU time (minutes) } \\
\text { Hospital stay (days) }\end{array}$ & $48.0 \pm 19.9$ & $50.4 \pm 12.0$ \\
$\begin{array}{l}\text { Perioperative complications } \\
\begin{array}{l}\text { Acetaminophen supplementation } \\
\text { (doses until discharge) }\end{array}\end{array}$ & $\begin{array}{l}1.7 \pm 1.9 \\
4.4 \pm 5.6\end{array}$ & $1.5 \pm 1.4$ \\
\hline
\end{tabular}

Notes: anly one patient had a non-zero FLACC pain score at discharge from the PACU. Data presented as mean \pm SD, with the exception of the perioperative complications data.

Abbreviations: FLACC, Faces, Leg, Activity, Crying, and Consolability scale; PACU, postanesthesia care unit. 
of children who tolerated oral feeds on the day of surgery or in the incidence of postoperative complications, including oxygen desaturation requiring supplemental oxygen, apnea, postoperative wound infection, persistent vomiting, and the need for surgical reexploration (Table 2). The majority of patients in both groups received postoperative acetaminophen supplementation (27 and 31 in the rectal and IV acetaminophen groups, respectively). Seven patients in the rectal group and three patients in the IV group did not require additional acetaminophen supplementation. No patient received IV acetaminophen postoperatively. The majority of postoperative acetaminophen was administered via the oral route although rectal acetaminophen was administered postoperatively in addition to oral acetaminophen in three patients in the IV group and two patients in the rectal group. There was no significant difference in the average number of supplemental acetaminophen doses between the two groups. During the postoperative course, the IV acetaminophen group averaged a total of 4.4 doses of supplemental acetaminophen versus 3.5 doses in the rectal acetaminophen group.

\section{Discussion}

The practice of anesthesiology involves choosing appropriate analgesic medications for a specific surgical patient population. The physiologic variability that exists among patients, including differences in gestational and chronological age, end-organ function, cardiopulmonary status, volume of distribution, and metabolic maturation, can affect drug pharmacodynamics and pharmacokinetics. Therefore, analgesic agents must be chosen judiciously, taking into account the patient's unique physiology. Opioids remain the primary analgesic regimen for perioperative pain. However, this class of analgesics is associated with dose-dependent adverse effects which may impact postoperative outcomes especially in neonates and infants. Alternative non-opioid analgesic agents exist, which may offer advantages to opioid monotherapy. ${ }^{11}$ The main theoretical advantage is the prevention of opioid side effects including constipation, nausea and vomiting, pruritus, respiratory depression, and opioid-induced hyperalgesia.

Opioid-related hypoventilation and apnea are of particular concern following anesthesia in neonates and infants undergoing pyloromyotomy for hypertrophic PS. Hypertrophic PS leads to projectile vomiting and hypochloremic metabolic alkalosis. Cerebrospinal fluid alkalosis may persist even after the correction of systemic metabolic alkalosis and $\mathrm{pH}$ normalization. ${ }^{5,6}$ Cerebrospinal fluid $\mathrm{pH}$ is one of the determinants of respiratory stimulus. ${ }^{5-7}$ This metabolic milieu along with hyperventilation (respiratory alkalosis), dehydration, use of neuromuscular blocking agents, the administration of inhalational anesthetic agents, and opioids during the anesthetic procedure can increase the risk of central apnea. The risk of central apnea is increased furthermore in premature infants who constitute approximately $12 \%$ of this population. Neonates are especially sensitive to the respiratory depressant effects of opioids related to the immaturity of the central respiratory control centers. ${ }^{8}$ Thus, an opioid-sparing technique is often advocated for these patients during pyloromyotomy. ${ }^{4}$ Nonsteroidal anti-inflammatory drugs (NSAIDs) are an important class of non-opioid analgesics which have been used for the treatment of pain for more than a century. ${ }^{12}$ Aspirin was introduced into the market by Bayer in 1899. The primary mechanism by which NSAIDs exert their effects is via inhibition of the arachidonic acid-cyclooxygenase pathways. ${ }^{13}$ This leads to a reduction in prostaglandin synthesis both peripherally at the site of injury and centrally in the spinal cord, decreasing inflammatory mediators and pain. IV ketorolac and IV ibuprofen are the two parenteral NSAIDs that are approved for use in the USA. However, given their adverse effect profile, it may be prudent to limit their use in the neonatal and infant population. ${ }^{14}$

Acetaminophen is a commonly used non-opioid analgesic with a well-established safety and tolerability profile. Hepatotoxicity is rare when used at therapeutic doses. It does not have the negative adverse effect profile that is associated with opioids (sedation, respiratory depression, constipation, and abuse potential). In addition, unlike NSAIDs, acetaminophen does not compromise renal function or increase the risk of bleeding. Acetaminophen is commercially available in many formulations. Rectal acetaminophen is a common route of administration. Although standard dosing of rectal acetaminophen is similar to oral dosing (10-15 mg/ $\mathrm{kg}$ ), more recent pharmacokinetic studies suggest that a larger initial loading dose $(40 \mathrm{mg} / \mathrm{kg})$ is required to achieve the desired plasma concentration. ${ }^{15}$ The preemptive administration of rectal acetaminophen has been shown to lower pain scores and decrease the need for rescue analgesia in patients, ranging from 7 years to 15 years of age, in various surgical procedures. ${ }^{16,17}$ However, due to its unpredictable systemic absorption, rectal acetaminophen's efficacy has been debated in medical literature. ${ }^{18}$ IV acetaminophen was approved by the US Food and Drug Administration in November 2010 for the management of mild-to-moderate pain, as an adjunct to opioids for the management of moderate-to-severe pain, and the reduction in fever. As compared with peak acetaminophen plasma concentrations following oral (45-60 minutes) and rectal administration (4 hours), IV acetaminophen results in 
rapid peak plasma concentrations at 15 minutes following infusion and an analgesic effect as quickly as 5 minutes with a duration of action up to 4 hours. ${ }^{19}$ Information regarding IV acetaminophen use and dosing strategies for neonates and infants is limited. Acetaminophen clearance reaches $84 \%$ of mature values by 1 year of age; however, clearance is decreased in neonates when compared with adults. ${ }^{20,21}$ The opioid-sparing effect of IV acetaminophen remains controversial. Sinatra et al reported a decreased need for rescue opioid therapy and a reduction in 24-hour total opioid consumption in adults undergoing major orthopedic surgery. ${ }^{22}$ Dashti et al and Korpela et al reported opioid-sparing effects with either rectal or IV administration. ${ }^{16,17}$ However, Hiller et al noted no effect on total opioid needs with IV acetaminophen after major spine surgery in children and adolescents. ${ }^{23}$

Our preliminary data suggest that there is no advantage with the use of IV acetaminophen over rectal acetaminophen for patients undergoing laparoscopic pyloromyotomy. The only variable for which there was a statistically significant difference between the two groups was acetaminophen dose $(P<0.0001)$. Patients who received IV acetaminophen had smaller doses (milligrams/kilograms) than patients who received acetaminophen rectally. The two groups did not differ significantly in PACU time, pain scores, acetaminophen supplementation, time to hospital discharge, time to oral feeding time, or perioperative complications. It may be that the inability to detect a difference relates to the fact that postoperative pain following laparoscopic pyloromyotomy is relatively mild and that effective analgesia can be achieved solely with the infiltration of a local anesthetic agent into the surgical site.

The limitations of this retrospective study include the nonrandom distribution of patients into the two groups. In addition, as noted earlier, additional data are needed regarding acetaminophen pharmacokinetics in this age group. The average IV acetaminophen dose was $8.6 \mathrm{mg} / \mathrm{kg}$ in the patients. Maintenance dosing ranging from $7.5 \mathrm{mg} / \mathrm{kg}$ to $10 \mathrm{mg} / \mathrm{kg}$ has been suggested, with some sources suggesting a loading dose of up to $20 \mathrm{mg} / \mathrm{kg}$ irrespective of age. However, regardless of the dose and the route of administration, the average pain scores were $<1$ in the PACU and all patients except for one had a pain score of 0 at the time of discharge, suggesting that effective analgesia was achieved in both groups. Another limitation of this retrospective study is the lack of uniform pain score documentation once on the hospital floor. Pain assessment on the floor typically occurs every 4 hours; however, some data were missing during our retrospective review.
IV acetaminophen has a clear benefit in patients who cannot tolerate acetaminophen via oral or rectal routes. However, outside of this scenario, the advantage of IV acetaminophen over other formulations is unclear. From a pharmacokinetics standpoint, IV acetaminophen may result in an earlier and higher peak plasma concentration. However, the clinical difference remains to be determined depending on the clinical scenario and the type of surgical procedure.

\section{Conclusion}

In summary, for patients undergoing laparoscopic pyloromyotomy, our preliminary data suggest that there is no clinical advantage with the use of IV compared with rectal acetaminophen. Pain scores, acetaminophen supplementation, PACU discharge time, length of hospital stay, and nonsurgical complications were similar in both groups (IV versus rectal acetaminophen). Given the superficial nature of the surgical incision sites, infiltration with a local anesthetic agent may play a bigger role in controlling postoperative pain than the acetaminophen formulation.

\section{Disclosure}

The authors report no conflicts of interest in this work.

\section{References}

1. St. Peter SD, Ostlie DJ. Pyloric stenosis: from retrospective analysis to prospective clinical trial-the impact on surgical outcomes. Curr Opin Pediatr. 2008;20:311-314.

2. Applegate MS, Druschel CM. The epidemiology of infantile hypertrophic pyloric stenosis in New York State, 1983 to 1999. Arch Pediatr Adolesc Med. 1995;149:1123-1129.

3. Li B, Chen WB, Wang SQ, Wang YB. Single-site umbilical laparoscopic pyloromyotomy in neonates less than 21-day old. Surg Today. 2015;45:29-33.

4. Bissonnette B, Sullivan PJ. Pyloric stenosis. Can J Anaesth. 1991;38:668-676.

5. Helmy MM, Tolner EA, Vanhatalo S, Voipio J, Kaila K. Brain alkalosis causes birth asphyxia seizures, suggesting therapeutic strategy. Ann Neurol. 2011;69:493-500.

6. Pappenheimer JR, Fencl V, Heisey SR, Held D. Role of cerebral fluids in control of respiration as studied in unanesthetized goats. Am J Physiol. 1965;208:436-450.

7. Abreu FA, Silva E, Macfadyen UM, Williams A, Simpson H. Sleep apnoea during upper respiratory infection and metabolic alkalosis in infancy. Arch Dis Child. 1986;61:1056-1062.

8. Andropoulos DB, Heard MB, Johnson KL, et al. Postanesthesia apnea in full-term infants after pyloromyotomy. Anesthesiology. 1994;80:216-219.

9. Habre W, Schwab C, Gollow I, Johnson C. An audit of postoperative analgesia after pyloromyotomy. Paediatr Anaesth. 1999;9: 253-256.

10. Voepel-Lewis T, Zanotti J, Dammeye JA, Merkel S. Reliability and validity of the face, legs, activity, cry, consolability behavioral tool in assessing acute pain in critically ill patients. Am J Crit Care. 2010;19:55-61.

11. Kehlet H. Multimodal approach to control postoperative pathophysiology and rehabilitation. Br J Anaesth. 1997;78:606-617. 
12. Vane JR. The fight against rheumatism: from willow bark to COX-1 sparing drugs. $J$ Physiol Pharmacol. 2000;51:573-586.

13. Vane JR, Botting RM. Mechanism of action of nonsteroidal antiinflammatory drugs. Am J Med. 1998;104:2S-8S.

14. Aldrink JH, Ma M, Wang W, Caniano DA, Wispe J, Puthoff T. Safety of ketorolac in surgical neonates and infants 0-3 months old. $J$ Pediatr Surg. 2011;46:1081-1085.

15. Birmingham PK, Tobin MJ, Fisher DM, Henthorn TK, Hall SC, Coté CJ. Initial and subsequent dosing of rectal acetaminophen in children: a 24-hour pharmacokinetic study of new dose recommendations. Anesthesiology. 2001;94:385-389.

16. Dashti GA, Amini S, Zanguee E. The prophylactic effect of rectal acetaminophen on postoperative pain and opioid requirements after adenotonsillectomy in children. Middle East J Anesthesiol. 2009;20:245-249.

17. Korpela R, Korvenoja P, Meretoja OA. Morphine sparing effect of acetaminophen in pediatric day-case surgery. Anesthesiology. 1999;91:442-447.

18. Anderson BJ, Woolard GA, Holford NHG. Pharmacokinetics of rectal paracetamol after major surgery in children. Paediatr Anaesth. $1995 ; 5: 237-242$.
19. Moller PL, Juhl GI, Payen-Champenois C, Skoglund LA. Intravenous acetaminophen (Paracetamol): comparable analgesic efficacy, but better local safety than its prodrug, propacetamol, for postoperative pain after third molar surgery. Anesth Analg. 2005;101:90-96.

20. Marier JF, Mouksassi S, Pan CC, et al. IV acetaminophen pharmacokinetics in children and adolescents is comparable to adults. Clin Pharmacol Ther. 2010;87:S39-S65.

21. Palmer GM, Atkins M, Anderson BJ, et al. IV acetaminophen pharmacokinetics in neonates after multiple doses. Br J Anaesth. 2008;101:523-530.

22. Sinatra RS, Jahr JS, Reynolds L, et al. Intravenous acetaminophen for pain after major orthopedic surgery: an expanded analysis. Pain Pract 2012;12:357-365.

23. Hiller A, Helenius I, Nurmi E, et al. Acetaminophen improves analgesia but does not reduce opioid requirement after major spine surgery in children and adolescents. Spine. 2012;37:E1225-E1231.
Journal of Pain Research

\section{Publish your work in this journal}

The Journal of Pain Research is an international, peer-reviewed, open access, online journal that welcomes laboratory and clinical findings in the fields of pain research and the prevention and management of pain. Original research, reviews, symposium reports, hypothesis formation and commentaries are all considered for publication.

\section{Dovepress}

The manuscript management system is completely online and includes a very quick and fair peer-review system, which is all easy to use. Visit http://www.dovepress.com/testimonials.php to read real quotes from published authors. 